\title{
Le Miroir, du théâtral au romanesque
}

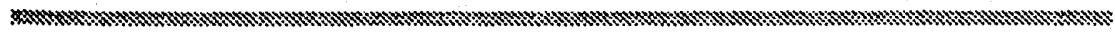

Olivier Tarnaud

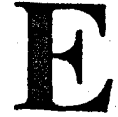

n une seule journée, Madame de Renal et l'Abbé Chelan vont exaucer les vœux de Julien Sorel. Après avoir traversé Verrières à cheval, il est appelé à Bray-le-Haut où doit avoir lieu une célébration religieuse en l'honneur du Roi de... Alors que ce demicr est sur le point d'arriver à l'Abbaye, l'évêque chargé de la cérémonie reste introuvable. Julien, motivé par l'arrogance des laquais du prélat, part à sa recherche et se retrouve bientôt dans une salle "extrêmement sombre" et "toute lambrissée de chêne noir" (Le Rouge et le noir: I, 18). Le passage que nous allons expliquer retrace les quelques minutes qui précedent les premières paroles de l'évêque et la réaction immédiate de Julien.

Le narrateur stendhalien décrit tout d'abord un décor simple, tout à fait thếtral. Il y place un miroir qui a plusicurs attributions: du miroir-spectateur qui temoigne de la mise en scène stendhalienne au miroir-abstraction qui revèle un moddle, en passant par le miroir-mensonge qui aide Julien à analyser les gestes de l'évêque, c'est tout le processus de formation de Julien qui est esquissé ici. En étudiant cette trifonctionnalité, nous verrons que ces quelques lignes sont à l'image du roman: nous comprendrons que chez Stendhal, la mise en scene est au service du romanesque, le décor au service de la fiction.

Examinons tout d'abord le cadre scénique décrit par le narrateur. Dans ce passage, c'est le dctail simple qui suscite le vrai et qui pernet au spectateur de visualiser la scene. Les couleurs, tout d'abord, sont très peu nombreuses. Au "noir" des lambris et de l'habit de Julien, s'oppose le rouge des "briques nues" (1) et la variante "violette" de la "robe" (4) du jeune homme qu'il aperçoit. Le blanc vient compléter ce tableau par l'intermédiaire du "plâtre encore tout blanc" (2) et du "surplis de dentelle" (5) de la robc ecclesiastique. Cette scène tricolore est d'une simplicitê extrême et le contraste existant entre ces trois 
couleurs permet au spectateur d'imaginer la salle dans laquelle elle se déroule.

Cet effet est complété par la précision suivante. Le narrateur nous avait auparavant indiqué "[qu']a l'exception d'une seule, les fenêtres avaient été murées". Il le répète ici en insistant sur l'unicite: "l'unique fenêtre" (3 et 13), c'est-à-dire une seule source de lumière qui permet néanmoins la distinction des formes. Elle représente aussi une frontière transparente entre le dehors et le dedans: c'est comme si le monde extérieur pouvait être témoin de cette scène, c'est comme si le lecteur pouvait regarder ce qui se passe à l'intérieur et témoigner. C'est un peu comme au thêâtre où le spectateur remplace le quatrième mur fictif de l'espace scénique.

Enfin, le narrateur termine la description de la salle en insistant sur la préseace du "miroir" (la répétition est telle que plusieurs synonymes sont utilisés). Il en devient l'objet central du décor. On ne peut s'empecher de voir dans cette insistance un effet au service du réalisme. "Ce meuble qui semblait étrange en un tel lieu" (6) est en fait placé la comme pour être témoin de la scène, le miroir est spectateur' tout comme la fenêtre même s'il est impliqué de façon différente puisqu'il se trouve au niveau des acteurs et non pas en surplomb comme la fenêtre.

Cette simplicité dans le détail et cette volonté de placer ça et là des témoins occulaires rend compte de l'effort du narrateur: il tient à authentifier ce qui se passe, a l'attester. Cette salle pourrait être une scène de thêatre où la fenêtre est spectatrice, ou le miroir est témoin de l'action des protagonistes. Pourtant. au réalisme de la scène s'adjoint le jeu des acteurs que le narrateur veut "décoriquer".

Ce "miroir ... en acajou" (4) qui ajoute au rouge du décor, est d'abord utilisés par "le jeune homme [qui] ... donn[e] gravement des bénédictions d[e] [son] côté ..." (8). Julien en est même surpris pusqu'il cherche à connaîtue la signifi[cation] de cette "cérémonie préparatoire" (9). Ces "bénédictions exécutées lentement mais en nombre infini" (14) sont revélatrices: "[1]e jeune prêtre" (10) répcte une attitude, il veut [ap]prendre un rôle et le miroir apparait alors comme un outil de transformation.

Grâce à ce miroir, le jeune homme s'approprie un masque. Le narrateur explique à plusieurs reprises que Julien pouvait distinguer son "air irrite" (T). son "air fâche" (16) : en fait, il s'exerce comme pour jouer un rôle. Cette anitude relève de son devoir de paraître.

Elle s'oppose à celle de Julien. N'oublions pas qu'il s'est senti blessé par l'attitude de l'entourage de l'évêque, il craint d'ailleurs un autre revers: - Tce jeune prêtre] sera insolent comme les laquais" (11), "[Julien] était froisśs d'avance par les mots durs qu'on allait lui adresser." (20-21). Pourtant, il est déterminé et, tout comme l'évêque, animé par un “devoir”, celui "de parler" (19). En revanche, il est emporte par son élan et sa spontanéité. Son naurel s'oppose a l'attitude artificielle de l'homme au miroir. C'est pourtant au 
moment où il se doute de l'importance de l'homme qu'il manque d'assurance: il est "arrêt[દ] par la richesse du surplis gami de dentelles." (16-17) L'apparence de l'autre atteint sa volonté alors qu'il s'approche de la vérité et qu'il croit reconnaître les signes distictifs, "la richesse" (16), de l'importance sociale. L'habit et le masque de l'homme ont agi sur le naturel de Julien qui s'estompe peu à peu.

C'est alors qu'intervient le troisième attribut du miroir. La concomittence est frappante: c'est lorsque le jeune homme se "retourn[e]" (22), après avoir aperçu Julien dans "la psyche", (et non après avoir entendu le bruit des éperons!), qu'il "quitt[e] subitement [son] air fâché." (22 et 23). Le miroir apparaît donc comme un instrument du mensonge, de l'hypocrisie: le prélat, cessant de se regarder dans le miroir, abandonne son air forcé au profit d'un air naturel.

Pourtant, bien que le jeune homme se soit retourné, il n'en est pas moins resté sous la fenêtre et cette image d'un homme dans la lumière fait figure de révelation pour Julien. Cette lumière indique à Julien la voie à suivre, "la croix pectorale" (26) devenant du même coup le symbole de la réussite. ce signe de nature religieuse lui permet de se situer dans la société, il guidera son destin.

En effet, on ne peut se tromper sur le sentiment de Julien: “[il] resta stupéfait" (25). La surprise est totale et la revélation sc transforme en admiration lorsqu'il se figure immédiatement la distance temporelle qui le sépare de la position sociale de l'évêque qui a "tout au plus six a huit ans de plus que [luil" (27). Le narrateur ne nous laisse pas le temps de nous interroger sur le contenu de ses pensces. Julien a choisi la voie tracée par la lumière et le miroir, celle de l'église et de son faste puisqu'il "eût honte de ses éperons" (28), signe distinctif du cavalier et par extension de l'armée. Cette citation est d'ailleurs très puissante: clle permet au lecteur de visualiser Julien et de rentrer dans son âme. Elle est aussi tout à fait symbolique puisque sous l'habit noir apparait sa réelle ambition qu'il n'a pas eu le temps de cacher.

Le miroir, son jeu et ses utilisations ont permis au narrateur de dessiner tout le contraste social de Julien. Par le jeu du double, par l'attitude de l'évêque, Julien a compris quel "devait" être son avenir. Ce verbe est utilisé a dessein car l"idée de forcer sa nature pour parvenir est la leçon de ce passage. On doit s'approprier un masque si l'on veut séduire son entourage: "l'art de plaire est l'art de tromper". ${ }^{1}$ Ces quelques lignes sont décisives pour la suite du roman. Encore hésitant quant à son futur proche, alors qu'il a eu la possibilité de passer "son bel habit bleu" de cavalier et celui, "râpe", de prêtre dans la même journée, Julien a vu la magnificence qui entoure un évêque et a fait son choix. Grâce au cadre simple et rigoureux du décor au milieu duquel le narrateur fait troner un miroir-spéctateur qui permet la thếtralisation, le lecteur peut visualiser la scène. Le réalisme du décor est bien chez Stendhal au service de la fiction romanesque. 
Notes

${ }^{1}$ Le Journal de Stendhal. Paris: Gallimard, 1955. 778. 

